\title{
Breast Cancer Management during Coronavirus Disease 2019 Pandemic: A Literature Review
}

\author{
Andree Kurniawan ${ }^{1,2, \odot ~ D e v i n a ~ A d e l l a ~ H a l i m ~}{ }^{3, \odot}$ Angela Giselvania ${ }^{4, \odot} \quad$ Noorwati Sutandyo ${ }^{5, \odot}$ \\ Sonar Soni Panigoro ${ }^{6}$ Asri Adisasmita ${ }^{7}$ Ratna Djuwita Hatma \\ ${ }^{1}$ Department of Internal Medicine, Faculty of Medicine, Pelita \\ Harapan University, Tangerang, Indonesia \\ ${ }^{2}$ Clinical Epidemiology, Faculty of Public Health, Universitas \\ Indonesia, Depok, Indonesia \\ ${ }^{3}$ Faculty of Medicine, Pelita Harapan University, Tangerang, \\ Indonesia \\ ${ }^{4}$ Department of Radiation Oncology, Dr. Cipto Mangunkusumo \\ Hospital, Faculty of Medicine, Universitas Indonesia, Jakarta, \\ Indonesia \\ ${ }^{5}$ Hematology and Medical Oncology, Department of Internal \\ Medicine, Dharmais Cancer Center Hospital, Faculty of Medicine, \\ Universitas Indonesia, Jakarta, Indonesia \\ ${ }^{6}$ Department of Surgical Oncology, Dr. Cipto Mangunkusumo \\ Hospital, Faculty of Medicine, Universitas Indonesia, Jakarta, \\ Indonesia \\ ${ }^{7}$ Department of Epidemiology, Faculty of Public Health, Universitas \\ Indonesia, Depok, Indonesia \\ Asian J Oncol 2021;7:52-59. \\ Address for correspondence Andree Kurniawan, MD, FINASIM, \\ Department of Internal Medicine, Faculty of Medicine, Pelita \\ Harapan University, Boulevard Jendral Sudirman Karawaci, \\ Tangerang, Banten 15811, Indonesia \\ (e-mail: andree.kurniawan@uph.edu).
}

\begin{abstract}
Keywords

- breast neoplasms

- COVID-19

- drug therapy

Introduction Breast cancer is the most prevalent cancer found in women. The World Health Organization declared the coronavirus disease 2019 (COVID-19) as a public health emergency of international concern. Delaying treatment was associated with mortality. The aim is to evaluate breast cancer management during the pandemic.

Methods We searched articles evaluating the management of breast cancer in the midst of COVID-19 outbreak, by searching in PubMed, PubMed Central, and Oncology Society related websites. We only included articles discussing adult breast cancer management in COVID-19 era. The two reviewers screened the titles and abstracts.

Results Around 124 articles were found through the searching process. Eight observational trials, two review articles, six guidelines or recommendations, and one letter to the editor were included in final review. It was concluded that breast cancer posed a risk to contract COVID-19. Newly suspected breast cancer could be deferred for screening or diagnosis, except for special breast cancer cases with bleeding, invasive, and complicated tumors. The benefit of radiotherapy option in every case should be carefully weighed against the risk of infection. Oral chemotherapy and hormonal therapy drugs were preferred to be given than intravenous chemotherapy. The scheduled chemotherapy could be adjusted to reduce hospital visit. Each breast cancer patient is different, so they should be discussed in multidisciplinary team consisting of surgical, radiation, and medical oncologists, pathologist, radiologist, palliative care people, nurses, nutritionist, and others related to the field. Conclusion The International Oncology Society has released recommendations for breast cancer during COVID-19 pandemic but there is still questionable-quality evidence.
\end{abstract}

published online January 27, 2021
DOI https://doi.org/

$10.1055 / \mathrm{s}-0040-1722809$ ISSN 2454-6798.
(C) 2021. Spring Hope Cancer Foundation \& Young Oncologist Group of Asia. This is an open access article published by Thieme under the terms of the Creative Commons Attribution-NonDerivative-NonCommercial-License, permitting copying and reproduction so long as the original work is given appropriate credit. Contents may not be used for commercial purposes, or adapted, remixed, transformed or built upon. (https://creativecommons.org/licenses/by-nc-nd/4.0/). Thieme Medical and Scientific Publishers Pvt. Ltd. A-12, 2nd Floor, Sector 2, Noida-201301 UP, India 


\section{Introduction}

Breast cancer is the most prevalent cancer found in women with an estimated number of new cases in the Europe community being 404,920 , while its estimated age-adjusted annual incidence is $144.9 / 100,000$. Worldwide, in 2018, the incidence of breast cancer in female was up to 2.1 million. ${ }^{1}$ In the same year in Asia, newly confirmed cases kept rising with an estimated increment of more than 900,000 cases, constituting $43.6 \%$ of the newly reported breast cancer cases worldwide. On the other hand, its breast cancer death comprised about half of worldwide mortality, reaching more than 300,000 deaths. $^{2}$

The World Health Organization declared the Coronavirus disease 2019 (COVID-19) as a public health emergency of international concern. The newly discovered disease is a challenge for health care professionals. The full impact of severe acute respiratory syndrome-coronavirus 2 (SARS-CoV2) in our health systems is yet to come and will be prolonged. Global confirmed cases of COVID-19 had reached more than 48.5 million, with more than 1.2 million deaths, while Indonesia has reported 429,574 cases with 14,442 deaths, as of November 6, 2020. ${ }^{3}$

Timing of surgery after neoadjuvant chemotherapy, especially in estrogen receptor positive (ER+)/human epidermal receptor 2 positive (HER2+) patients if delayed more than 8 weeks, would impact on decreased overall survival and disease-free survival. ${ }^{4}$ Another study found that delaying breast cancer treatment of even a single modality will impact on higher breast cancer-specific mortality and all-cause mortality. ${ }^{5}$

There is still limited data to recommend care for breast cancer patients in the midst of COVID-19 outbreak, resulting in fear of delay in treatment and impact on the overall survival of patients. The aim of this study is to know the best recommendation for breast cancer care during COVID-19 outbreak.

\section{Methods}

\section{Search Strategies}

A comprehensive search of the literature was conducted by searching in PubMed and PubMed Central databases, and for guidelines or recommendation by international societies of surgical oncology, radiation oncology, and medical oncology, from January 2020 to April 2020, with keyword combinations of the medical subject headings (MeSH) on April 9, 2020. The keywords "breast neoplasm" AND "COVID-19" AND "management" were used in search engine. Management of adult breast cancer patients through surgery, chemotherapy, and radiotherapy (RT) were the inclusion criteria. Articles in non-English language were excluded. Firstly, we searched the titles and abstracts. Then, we evaluated the full text whether it discussed breast cancer care in COVID-19 times.

\section{Results and Discussion}

\section{Literature Search}

We discovered 124 articles through the literature search strategy. The reviewing process of literature based on systematic reviews and meta-analysis (e.g., PRISMA: preferred reporting items for systematic reviews and meta-analyses) can be seen in -Fig. 1. The two reviewers (A.K. and D.A.H.) screened the title and abstracts for 124 articles. Finally, we only included articles mainly discussing breast cancer management in adults during COVID-19 pandemic. There were eight observational trials, two review articles, six guidelines or recommendations, and one letter to the editor. The diagram of search literature can be seen in $\boldsymbol{- F i g . ~} \mathbf{1}$.

\section{Risk of COVID-19 Infection in Cancer Patients}

From the longitudinal COVID-19 infection data in China reported by Liang et $\mathrm{al}^{6}{ }^{6}$ the incidence of cancer in the COVID-19 population seems to be higher than in the overall Chinese population. Breast cancer was found in 3 of 18 cancer patients (two in remission and one no information). Cancer patients were older (63.1 vs 48.7 years) and smokers (22\% vs $7 \%$ ). At diagnosis of COVID-19, patients presented more frequently with tachypnea ( $47 \%$ vs $23 \%$ ) and with a more severe computed tomography (CT) pattern of infection (94\% vs 75\%). Cancer groups are more likely to develop more severe illness or death $(50 \%$ vs $16 \% ; p=0.0008)$, even worse in those undergoing active treatment ( $75 \%$ vs $43 \%) .{ }^{6}$ After adjusting for the variables age and smoking in cancer patients, the results showed an odds ratio $(\mathrm{OR})$ for more severe complications $(\mathrm{OR}=5.34$; $95 \%$ confidence interval $=1.8-16.18 ; p=0.00026$ ). The risk seemed to be independent of cancer types.?

\section{What Oncologist Should Do?}

Discussing the possible options for health care professionals on how to deal with patients with recent diagnoses/suspected breast cancer or with ongoing treatment within the recent COVID-19 pandemic context should be done in a multidisciplinary team (MDT) meeting. Several scenarios should be considered for breast cancer management according to the diverse pandemic scenarios in different countries. ${ }^{8}$

\section{Preparation}

Test all patients planned to receive elective surgery and emergency surgery for COVID-19, like using rapid tests. The decision needs to comply with local test availability, and choosing the proper test needs to rely on proper timing to obtain valid test results. ${ }^{8}$

\section{Screening and Diagnosis}

Breast cancer screening, with mammographic use, should be postponed until the outbreak is over. Symptomatic cases that need diagnostic procedure should be managed according to local availability. On the other hand, considering the burden 


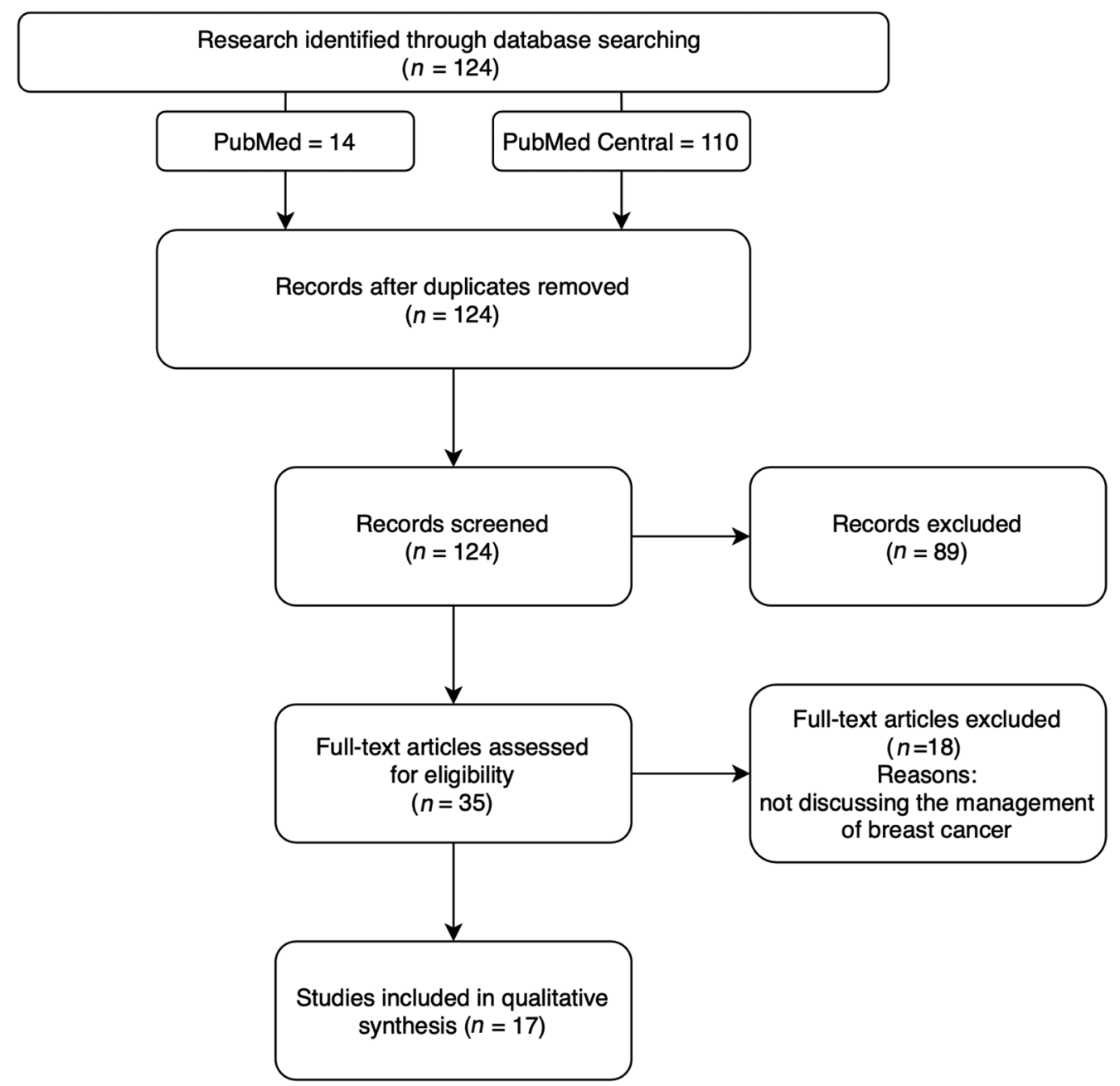

Fig. 1 PRISMA (preferred reporting items for systematic reviews and meta-analyses) search strategy.

on clinical outcomes, regular evaluation should be performed in patients with unusual symptoms and signs. ${ }^{8}$

Initial breast cancer work-up should be conducted only in patients with node-positive breast cancer. Magnetic resonance imaging should only be done in COVID-19 negative patients due to the risk of device contamination. CT scan should be avoided whenever possible because the prioritization is for COVID-19 positive patient evaluation. Other imaging use, for instance echocardiography monitoring, was considered to be postponed if no cardiac symptoms and no previous reduction in ejection fraction were observed. Alternatively, troponin and brain natriuretic peptide laboratory checks from blood samples can be done. ${ }^{8}$

Outpatient consultation should be changed to telemedicine consultation. Appointments should be prioritized according to urgency using high, medium, and low-priority stratification. ${ }^{8}$ Further information about stratification can be retrieved from - Table $\mathbf{1}$.

\section{Surgery}

Ideally, team members must be reduced to lessen the possibility of contacts and allow for replacement in case of getting contracted. All booking procedures per day should be reduced, regarding the time needed to disinfect carefully. Most procedures should be technically contraindicated or need to be replaced. ${ }^{8}$

All decisions to proceed with the surgical procedure should be discussed in MDT meeting, which could be held through teleconference platform. The results should be further deliberated with the patient and should depend on their desire to take the final decision.

Before initiating invasive procedure, such as surgery, ideally all patients should be screened for COVID-19, although asymptomatic. When the presence of SARS-CoV2 is detected, the patients should be treated as COVID-19 patient first while the procedure and/or treatment can be resumed afterward. ${ }^{9}$ 
Table 1 Stratification of outpatient consultation appointments into priority groups ${ }^{8}$

\begin{tabular}{|c|c|c|}
\hline Priority & Interval until surgery & Recommendation \\
\hline Urgency & $<2$ weeks & $\begin{array}{l}\text { - Patients with significant tumor burden not responding or progressing under PST } \\
\text { - Pregnant patients } \\
\text { - Patients with complicated locally advanced tumor }\end{array}$ \\
\hline High priority & 2-4 weeks & $\begin{array}{l}\text { - Patients with early isolated locoregional recurrence (within } 48 \text { months from pri } \\
\text { mary treatment) } \\
\text { - High-risk patients with contraindications to PST, if younger than } 40 \text { years or } \\
\text { node-positive, or with disease showing biological features of aggressiveness }\end{array}$ \\
\hline Medium priority & 4-8 weeks & $\begin{array}{l}\text { - Patients treated with PST (ideally at a maximum of } 4-6 \text { weeks after treatment } \\
\text { completion) } \\
\text { - Premenopausal patients with small }(<2 \mathrm{~cm}) \text { node-negative tumors }\end{array}$ \\
\hline Low priority & $>8$ weeks & $\begin{array}{l}\text { - Ductal carcinoma in situ (However, high-grade ER-negative or very extensive DCIS } \\
\text { and/or with a palpable lump or extensive microcalcifications might fall into the } \\
\text { intermediate priority category, based on individual consideration of each case) } \\
\text { - Postmenopausal patients with luminal A-like cancer; in these women, primary } \\
\text { endocrine therapy could be initiated and surgery could be postponed }\end{array}$ \\
\hline
\end{tabular}

Abbreviations: DCIS, ductal carcinoma in situ; ER, estrogen receptor; PST, primary systemic therapy.

All nonurgent and benign surgery indications should be deferred. Outpatient surgery should be offered to indicated patients whenever possible. Oncoplastic surgery, which needs prolonged hospitalization, should be minimized. In specific cases, immediate breast reconstruction was to be considered if the procedure remained simple and speeded recovery. However, microsurgery procedure should be postponed because of high resources required. ${ }^{8}$

\section{Recommendation for Surgery in Breast Cancer}

In recent weeks there were recommendations from the cancer working groups and country experiences in caring breast cancer patients in the COVID-19 era. Further details of surgical therapy priority in breast cancer have been provided in - Table 2 .

\section{Radiotherapy}

The role of RT in breast cancer causes significant impact on reducing locoregional recurrences and improving survival. However, in particular patients it might have no survival implications, for instance in ductal carcinoma in situ cases. The use of breast RT has to be thoughtfully evaluated in terms of its risk toward infection, in the middle of the pandemic. ${ }^{11}$

Recommendations for RT for breast cancer in the COVID-19 pandemic include the changes in framework for patient care, ${ }^{12}$ such as use of remote visits/teleconsultations, patient prioritization, method of radiation, avoidance, delay or omission of RT, and the use of hypofractionation techniques.

\section{Teleconsultations}

Teleconsultations, using simple call or video calls, should be implemented to replace outpatient visit when possible. ${ }^{12}$ Telehealth technology can also be used upon availability, depending on local institutional resources and legal aspects. For clinic patients or those who are coming for their RT sessions, special strategy should be arranged. Patients should come only at their time of appointment and should spend the waiting time elsewhere until their scheduled time. Visitor numbers should be cut down to allow physical distancing in the waiting room. Patients should not be not allowed to bring more than one companion. Laboratory testing should be performed with minimal contact to staff or other patients, and to avoid a busy hospital, possibly with a drive through system. Posttreatment routines, like tumor marker testing, can safely be postponed for up to 3 months. ${ }^{12}$

\section{Patient Prioritization}

Under extreme circumstances, it is essential to make priority setting for patients who might be given RT for their survival benefit. Some tiers of elevated priority have been prepared based on available evidence and clinical judgment. This priority setting should be considered by the physician regarding the patient age, comorbidities, risk of exposure, and benefit prediction of RT. ${ }^{8}$ Further details are available in - Table 3.

\section{Breathing Control}

Active breathing control technique in breast RT is not recommended during the COVID-19 pandemic, since it may increase the risk of aerosol contamination. Additionally, consideration should be taken to minimalize the use of device requiring decontamination. To reduce cardiac toxicities with no supplementary equipment and the risk of infection, deep inspiratory breath-hold techniques with voluntary breath hold might support. ${ }^{13}$

\section{Omission and Delay of Radiotherapy}

Breast RT can be considered to be omitted in certain settings when the effect on survival is not very significant, such as in patients with noninvasive disease or older patients with low-risk disease who have minimal survival benefit from breast RT and much greater risk of mortality from COVID-19. ${ }^{13}$

Experts in several countries in the world discussed and made several recommendations for RT in breast cancer patients 
Table 2 Priorities for breast cancer: surgical oncology ${ }^{10}$

\begin{tabular}{|l|l|}
\hline Priority & Recommendation \\
\hline High & $\begin{array}{l}\text { - Breast cancer surgery complication with bleeding or indication to incision and drainage of a breast abscess and/ } \\
\text { or hematoma }\end{array}$ \\
\hline & $\begin{array}{l}\text { - Complications of reconstructing surgery, for instance, ischemia } \\
\text { - Surgery in patients who have completed neoadjuvant chemotherapy-based treatment (or cases with progression } \\
\text { - Surgery in patients with invasive cancer after discussed in MDT } \\
\text { - Breast cancer surgery during pregnancy (MDT decision should be according to the stage and biology) }\end{array}$ \\
\hline Medium & $\begin{array}{l}\text { - Excision of malignant recurrence (depending on phenotype and extent) } \\
\text { - Clinically low-risk primary breast cancer (e.g., stage I/II ER-positive/PR-positive/HER2-negative, low grade/low } \\
\text { - Discordant biopsies likely to be malignant }\end{array}$ \\
\hline Low & $\begin{array}{l}\text { - Excision of benign lesions and duct excision (fibroadenomas, atypia, papilloma) } \\
\text { - Surgery of noninvasive breast cancer (in situ), except for extended high-grade DCIS } \\
\text { - Biscordant biopsies likely to be benign } \\
\text { - Prophylactic surgery for asymptomatic high-risk patients }\end{array}$ \\
\hline
\end{tabular}

Abbreviations: DCIS, ductal carcinoma in situ; ER, estrogen receptor; HER2, human epidermal receptor 2; MDT, multidisciplinary tumor board meeting; $\mathrm{PR}$, progesterone receptor.

Table 3 Prioritization of radiation for breast cancer based on treatment indication ${ }^{8}$

\begin{tabular}{|l|l|}
\hline High priority for breast RT & - Inflammatory breast cancer \\
& - Residual node positivity after NAC \\
& - Four or more positive nodes (N2) \\
& - Recurrent disease \\
& - Node-positive triple-negative BC \\
- Extensive LVI
\end{tabular}

Abbreviations: BC, breast cancer; ER, estrogen receptor; LVI, lympho-vascular invasion; NAC, neoadjuvant chemotherapy; NOS, no other specified; RT, radiotherapy.

during COVID-19 pandemic. RT should be omitted for patients aged 65 years and older (or with relevant comorbidities in younger age) with invasive breast cancer that are up to $30 \mathrm{~mm}$ with clear margins, grades 1 to 2, ER+, HER2-negative, and node negative planning for treatment with endocrine therapy.

\section{Hypofractionation}

Adjuvant breast RT has been established as part of standard treatment with normal fractionated regimen of $50 \mathrm{~Gy}$ in 25 fractions daily over 5 weeks with or without a boost of 10-16 Gy in 5 to 8 fractions. Hypofractionation is possible in breast RT with strong supporting data. In the COVID-19 pandemic era, it is recommended to use hypofractionation scheme for all breast/chest wall and nodal RT, such as 40 Gy in 15 fractions over 3 weeks. ${ }^{14}$ Extreme hypofractionation scheme can be considered for patients with node-negative tumors that do not require a boost, as in hypofractionated breast RT for 1-week versus 3-week (FAST Forward) trial, with options including 28 to $30 \mathrm{~Gy}$ in 5 fractions weekly over 5 weeks or 26 Gy in 5 fractions daily over 1 week, respectively. ${ }^{14}$

In order to reduce the number of fractions and/or complexity of treatment, a boost in breast RT should be considered to be omitted whenever possible, unless in those with significant risk for local relapse, that is, 40 years old and under, or over 40 years with significant risk factors for local relapse. Nodal RT can be omitted in postmenopausal women requiring whole breast RT following sentinel lymph node biopsy and primary surgery for T1, ER+, HER2-negative, and G1 to 2 tumors with 1 to 2 macro metastases..$^{15-17}$ Further indications of omitting RT treatment are provided in - Table 4 . 
Table 4 Radiotherapy treatments that may be omitted in breast cancer ${ }^{13,15}$

\begin{tabular}{|l|l|l|}
\hline Subsite or classification & Modality & Comments and evidence \\
\hline $\begin{array}{l}\text { - Breast conservation } \\
\text { - DCIS }\end{array}$ & $\begin{array}{l}\text { Omission of radiotherapy to the whole } \\
\text { breast }\end{array}$ & $\begin{array}{l}\text { No survival benefit; small benefit in } \\
\text { locoregional recurrence }\end{array}$ \\
\hline $\begin{array}{l}\text { - Invasive disease } \\
\text { - Low risk, older patients }\end{array}$ & $\begin{array}{l}\text { Omission of radiotherapy to the whole } \\
\text { breast }\end{array}$ & $\begin{array}{l}\text { Endocrine therapy only sufficient in >70 } \\
\text { >65 in PRIMEIl trial) }\end{array}$ \\
\hline $\begin{array}{l}\text { - Invasive disease } \\
\text { - Genomic profile, low risk }\end{array}$ & $\begin{array}{l}\text { Omission of radiotherapy to the whole } \\
\text { breast }\end{array}$ & $\begin{array}{l}\text { LUMINA, IDEA, PRECISION, PRIMETIME } \\
\text { trials ongoing (caution outside of trial) }\end{array}$ \\
\hline $\begin{array}{l}\text { - Age } \geq 50, \text { ER+, HER2-breast cancer with- } \\
\text { out other adverse pathologic features }\end{array}$ & Omission of boost radiotherapy & No survival benefits \\
\hline $\begin{array}{l}\text { - Postmastectomy } \\
\text { - T1-2 N1 (node + breast cancer) }\end{array}$ & Omit radiotherapy & NSABP B-51/RTOG 1304 trials ongoing \\
\hline
\end{tabular}

Abbreviations: DCIS, ductal carcinoma in situ; ER, estrogen receptor; HER2, human epidermal receptor 2.

Delay in RT treatment may be safely done for ER+ breast cancer patient for up to 5 months if they are established on endocrine therapy and have received prior chemotherapy. ${ }^{16,17}$

\section{Systemic Therapy}

The objective of systemic therapy is to ensure appropriate cancer care and to protect patients with cancer from the risk of COVID-19 infection. ${ }^{8}$

We should minimize the presence of cancer patients at the hospital. Patients should be encouraged to be managed at home by telemedicine and phone calls. Oral chemotherapy and hormonal therapy drugs should be preferred to intravenous drugs. Other alternatives such as intravenous and subcutaneous anticancer agents can be made available at home. The schedule of chemotherapy could be adjusted to decrease hospital visit, for example every 3 weeks, compared to weekly basis. Patients with slowly evolving metastatic cancer can break temporarily. All the decisions should be discussed by the MDT and shared with patients and their families. ${ }^{18,19}$

The preventive measures in hospitals should be improved. Daily phone calls to check symptoms of COVID-19 in patients, who are planned for hospital treatment in the following day, should be done regularly. Separation measures in hospital must be implemented, for example ensuring appropriate space between seats. Caregivers should not be not allowed for all in- and outpatients except in selected cases, for instance for children who need continuous assistance. Surgical masks and handwashing with a hydro-alcoholic solution should be provided to all patients and staff at the hospital entrance. MDT meetings should be done in a virtual setting or internally. ${ }^{18,19}$

The criteria for patient admission to hospital for systemic therapy should depend on curative or palliative intent, patient age, and their life expectancy. The priority for patient admission to hospital for systemic therapy should be set in the following order: (1) patient with curative intent treatment, favoring those with less than or equal to 60 years or life expectancy $\geq 5$ years; (2) cancer patients with (a) noncurative intent and less than or equal to 60 years old, (b) 5 years life expectancy or more, or (c) both and in first-line; and (3) others with noncurative care, favoring those with quickly evolving and symptomatic metastatic cancer. ${ }^{8}$

\section{Recommendation for Systemic Therapy}

There is still limited study related to breast cancer management during the COVID-19 pandemic. In recent weeks, there were recommendations from the cancer working groups and country experiences in handling breast cancer patients during COVID-19. For further details on the priority of systemic therapy in early and metastatic breast cancer, see - Tables 5 and 6 .

\section{Supportive Care}

Home administration of supportive care should be implemented in all cancer patients. Patients were taught to do physical and recreational activities using web-platforms. Psychological support also could be provided by phone calls or other telemedicine measures. ${ }^{8}$

Medical rehabilitation should be postponed, especially several activities like use of nebulizer, cough assist manual, cough assist measures such as flutter and acapella, and cough exercise. Some conditions can be postponed such as spinal cord injury, acute neuromuscular disease, severe pain not relieved by optimal pain killers, severe mucus retention, dysphagia, and sudden loss of body function. ${ }^{22}$

\section{Clinical Trial}

Participation in clinical trials should be adjusted. Patients' accrual in ongoing trials should be limited and case-by-case accrual in trials providing new effective treatments to patients without valid standard alternatives should be discussed. On-study patients could be referred to other active centers in the case of issues due to COVID-19 reorganization. In-person visits should be replaced with telephone calls or other telemedicine visits, and also the implementation of drug shipment to patients should be ensured. Research nurses' visits should be replaced with phone calls or other telemedicine visit measures. ${ }^{8,21}$

\section{Conclusion}

This review was done in the middle of COVID-19 outbreak. From the literature search results, there is still paucity of evidence regarding the management of breast cancer in COVID-19 times. Several oncology societies have released 
Table 5 Priorities for breast cancer: medical oncology-early breast cancer ${ }^{20,21}$

\begin{tabular}{|c|c|}
\hline Priority & Recommendation \\
\hline High & $\begin{array}{l}\text { - Neoadjuvant and adjuvant chemotherapy for triple-negative breast cancer patients } \\
\text { - Neoadjuvant and adjuvant chemotherapy in combination with targeted therapy for HER2-positive } \\
\text { breast cancer patients } \\
\text { - Neoadjuvant and adjuvant endocrine therapy } \pm \text { chemotherapy for high-risk ER-positive/HER2-negative } \\
\text { breast cancer } \\
\text { - Completion of neoadjuvant chemotherapy (with or without anti-HER2 therapy) that has already been } \\
\text { initiated } \\
\text { - Continuation of adjuvant capecitabine treatment in high-risk triple-negative breast cancer patients, and } \\
\text { T-DM1 in high-risk HER2-positive breast cancer patients (in the post neoadjuvant settings) } \\
\text { - Continuation of treatment in the context of a clinical trial, provided patient benefits outweigh risks, with } \\
\text { possible adaptation of procedures without affecting patient safety and study conduct }\end{array}$ \\
\hline Medium & $\begin{array}{l}\text { - For postmenopausal women with stage } 1 \text { cancers, with low-intermediate-grade tumors, or lobular } \\
\text { breast cancers, endocrine therapy may be started first while surgery can be delayed } \\
\text { - For patients with low-risk genomic signatures/score, prefer endocrine therapy alone } \\
\text { - Ongoing adjuvant trastuzumab alone may be postponed by } 6-8 \text { weeks in patients at high risk of com- } \\
\text { plicated COVID-19 infection }\end{array}$ \\
\hline Low & $\begin{array}{l}\text { - Follow-up imaging, restaging studies, echocardiograms, ECGs, and bone density scans can be delayed if } \\
\text { patient is clinically asymptomatic or has clinical signs of response in the neoadjuvant settings }\end{array}$ \\
\hline
\end{tabular}

Abbreviations: COVID-19, Coronavirus disease 2019; ECGs, electrocardiograms; ER, estrogen receptor; HER2, human epidermal receptor 2.

Table 6 Priorities for breast cancer: medical oncology-metastatic breast cancer ${ }^{20,21}$

\begin{tabular}{|l|l|}
\hline Priority & Recommendation \\
\hline High & $\begin{array}{l}\text { - Early line chemotherapy, endocrine therapy, targeted therapy agents, or immune checkpoint inhibitors likely to } \\
\text { improve outcomes in metastatic disease (high priority to pertuzumab/trastuzumab plus chemotherapy in HER2- } \\
\text { positive breast cancer) } \\
\text { - Visceral crisis } \\
\text { - Continuation of treatment in the context of a clinical trial, provided patient benefits outweigh risks, with possible } \\
\text { adaptation of procedures without affecting patient safety and study conduct }\end{array}$ \\
\hline Medium & $\begin{array}{l}\text { - Second-, third-, and beyond third-line treatment when therapy may provide clinical benefit and impact on outcome } \\
\text { - Consider avoiding or delaying the addition of mTOR or PIK3CA inhibitors to endocrine therapy, particularly in elderly } \\
\text { patients with comorbidities }\end{array}$ \\
& $\begin{array}{l}\text { - Bone agent therapy (zoledronic acid, denosumab) not urgently needed for hypercalcemia, or not needed for pain } \\
\text { control and in patients who are otherwise not in need of coming to the hospital (for instance receiving oral chemo- } \\
\text { therapy or endocrine therapy); bone agents can be administered every } 3 \text { months } \\
\text { at lengthened intervals }\end{array}$ \\
\hline
\end{tabular}

Abbreviations: ECGs, electrocardiograms; HER2, human epidermal receptor 2; mTOR, mammalian target of rapamycin; PIK3CA, phosphatidylinositol-4,5-bisphosphate 3-kinase catalytic subunit alpha.

guidelines about the management of breast cancer patients. The guidelines are based on experiences of experts in breast cancer field. Till present time, the COVID-19 cases have kept increasing in the world. Hence, people who are involved in breast cancer management are working together hand in hand to solve the problem and give the best for patient care.

A group of experts from various specializations is required for the optimal care of breast cancer patients. The MDTs should regularly meet up to discuss every problematic breast cancer patient, especially who got infected with COVID-19. This meeting will allow all key oncology specialists to join the discussion on individual breast cancer patient. The MDT consists of surgical, radiation, and medical oncologists together with pathologist, pharmacist, radiologist, nurses, palliative care people, and nutritionists. ${ }^{23}$

Severalconsortiums of cancerandCOVID-19 registry ${ }^{24}$ have been formed, involving multinational oncology centers in the world. From the cancer registry, we can learn more about the management of cancer, especially breast cancer, in COVID-19 era.

Several international oncology societies have released the breast cancer recommendations during the COVID-19 pandemic but there is still questionable-quality evidence. In this difficult situation, all people who are involved in oncology field will learn together regarding cancer management in COVID-19 times. ${ }^{25}$ 


\section{Conflict of Interest}

None declared.

\section{Acknowledgments}

The authors would like to thank their students for the support in searching and evaluating the articles.

\section{References}

1 Cardoso F, Kyriakides S, Ohno S, et al; ESMO Guidelines Committee. Early breast cancer: ESMO Clinical Practice Guidelines for diagnosis, treatment and follow-up. Ann Oncol 2019;30(8):1194-1220

2 Park YH, Senkus-Konefka E, Im SA, et al. Pan-Asian adapted ESMO Clinical Practice Guidelines for the management of patients with early breast cancer: a KSMO-ESMO initiative endorsed by CSCO, ISMPO, JSMO, MOS, SSO and TOS. Ann Oncol 2020;31(4):451-469

3 World Health Organization. Weekly Operational Update on COVID-19. Geneva: WHO; 2020. Available at: https:// www.who.int/publications/m/item/weekly-operational-update-on-covid-19-6-november-2020. Accessed November 7, 2020

4 Suleman K, Almalik O, Haque E, et al. Does the timing of surgery after neoadjuvant therapy in breast cancer patients affect the outcome? Oncology 2020;98(3):168-173

5 Yung R, Ray RM, Roth J, et al. The association of delay in curative intent treatment with survival among breast cancer patients: findings from the Women's Health Initiative. Breast Cancer Res Treat 2020;180(3):747-757

6 Liang W, Guan W, Chen R, et al. Cancer patients in SARSCoV-2 infection: a nationwide analysis in China. Lancet Oncol 2020;21(3):335-337

7 Guan WJ, Ni ZY, Hu Y, et al; China Medical Treatment Expert Group for Covid-19. Clinical characteristics of Coronavirus disease 2019 in China. N Engl J Med 2020;382(18):1708-1720

8 European School of Oncology. Guidelines and pathways of breast cancer care during the COVID-19 pandemic. www.eeso.net. Accessed December 14, 2020

9 Curigliano G, Cardoso MJ, Poortmans P, et al; Editorial Board of The Breast. Recommendations for triage, prioritization and treatment of breast cancer patients during the COVID-19 pandemic. Breast 2020;52:8-16

10 American College of Surgeons. COVID-19 Guidelines for Triage of Breast Cancer Patients. Available at: https://www.facs. org/covid-19/clinical-guidance/elective-case/breast-cancer. Accessed December 14, 2020

11 Braunstein LZ, Gillespie EF, Hong L, et al. Breast radiation therapy under COVID-19 pandemic resource constraints-approaches to defer or shorten treatment from a comprehensive cancer center in the United States. Adv Radiat Oncol 2020;5(4):582-588
12 Zaorsky NG, Yu JB, McBride SM, et al. Prostate cancer radiation therapy recommendations in response to COVID-19. Adv Radiat Oncol 2020;5(4):659-665

13 Simcock R, Thomas TV, Estes C, et al. COVID-19: global radiation oncology's targeted response for pandemic preparedness. Clin Transl Radiat Oncol 2020;22:55-68

14 Coles CE, Aristei C, Bliss J, et al. International guidelines on radiation therapy for breast cancer during the COVID-19 pandemic. Clin Oncol (R Coll Radiol) 2020;32(5):279-281

15 ASTRO. COVID-19 Recommendations to Radiation Oncology Practices. Available at: https://www.astro.org/Daily-Practice/ COVID-19-Recommendations-and-Information. Accessed April 1,2020

16 Harris EE, Christensen VJ, Hwang WT, Fox K, Solin LJ. Impact of concurrent versus sequential tamoxifen with radiation therapy in early-stage breast cancer patients undergoing breast conservation treatment. J Clin Oncol 2005;23(1):11-16

17 Cecchini MJ, Yu E, Potvin K, D'souza D, Lock M. Concurrent or sequential hormonal and radiation therapy in breast cancer: a literature review. Cureus 2015;7(10):e364

18 Al-Shamsi HO, Alhazzani W, Alhuraiji A, et al. A practical approach to the management of cancer patients during the novel coronavirus disease 2019 (COVID-19) pandemic: an International Collaborative Group. Oncologist 2020; 25(6):e936-e945

19 Lambertini M, Toss A, Passaro A, et al. Cancer care during the spread of coronavirus disease 2019 (COVID-19) in Italy: young oncologists' perspective. ESMO Open 2020;5(2):e000759

20 European Society of Medical Oncology. ESMO management and treatment adapted recommendation in the COVID-19 era: breast cancer. Available at: https://www.esmo.org/guidelines/ breast-cancer/breast-cancer-in-the-covid-19-era. Accessed April 11,2020

21 National Comprehensive Cancer Network. Coronavirus disease 2019 (COVID-19) resources for the cancer care community. Available at: https://www.nccn.org/covid-19/. Accessed April 11, 2020

22 Putra HL, Tamin TZ, Paulus AFS, Sungkar E, Sapta ADI Rekomendasi pelayanan kedokteran fisik dan rehabilitasi di Indonesia terkait COVID-19. Ist edition. Perhimpunan dokter spesialis kedokteran fisik dan rehabilitasi Indonesia (PB PERDOSRI); 2020

23 Saini KS, Taylor C, Ramirez AJ, et al. Role of the multidisciplinary team in breast cancer management: results from a large international survey involving 39 countries. Ann Oncol 2012; 23(4):853-859

24 European society of Medical Oncology. ESMO-CoCare registry. Available at: https://www.esmo.org/covid-19-andcancer/collaborating-on-registries-studies-and-surveys/ esmo-cocare-registry. Accessed May 31, 2020

25 Kurniawan A, Lugito NPH, Baskoro BA, Halim DA. Management of cancer patients during coronavirus disease 2019 pandemic: a literature review. Indon J Cancer 2020;14(2):69-74 